\title{
ISOSPECTRAL POTENTIALS ON A DISCRETE LATTICE. III
}

\author{
THOMAS KAPPELER
}

\begin{abstract}
Continuing prior work of the author, spectral problems for a discretized version of the Schrödinger equation 'Laplacian plus potential' on the torus are considered. Spectral invariants are constructed and with their help isospectral sets of certain nongeneric potentials are determined.
\end{abstract}

\section{INTRODUCTION AND SUMMARY}

Continuing investigations of $[\mathrm{K} 1, \mathrm{~K} 2]$ we consider the discrete Schrödinger equation

$$
\begin{gathered}
\Delta_{\text {discrete }} U(x)+Q(x) U(x)=\lambda U(x) \quad\left(\text { on } \mathbf{z}^{d}\right), \\
U(x+l)=e^{i \alpha \cdot l} U(x) \quad(l \text { in } L),
\end{gathered}
$$

where $L$ denotes a lattice in $\mathbf{Z}^{d}$ of the form $N \mathbf{Z} \oplus \cdots \oplus N \mathbf{Z}(N \geq 2), Q$ denotes a $L$-periodic potential and $\alpha=\left(\alpha_{1}, \ldots, \alpha_{d}\right)$ in $\mathbf{R}^{d}$ is the so-called crystal momentum. Observe that $\alpha=0$ corresponds to periodic boundary conditions. We refer to (1.2) as the $\alpha$-periodic boundary condition. As in $[\mathrm{K} 1, \mathrm{~K} 2] \Delta_{\text {discrete }}$ is conveniently defined as $\Delta_{\text {discrete }} U(x)=\sum_{\|x-y\|_{1}=1} U(y)$ for $U: \mathbf{Z}^{d} \rightarrow \mathbb{C}$ with $\|x\|_{1}=\sum_{i=1}^{d}\left|x_{i}\right|$ for $x$ in $\mathbf{Z}^{d}$.

For the convenience of the reader, let us give a matrix representation of the operator (1.1)-(1.2), when restricted to a fundamental domain of $\Gamma$, with respect to a suitably chosen canonical basis, in the case where $Q=0$.

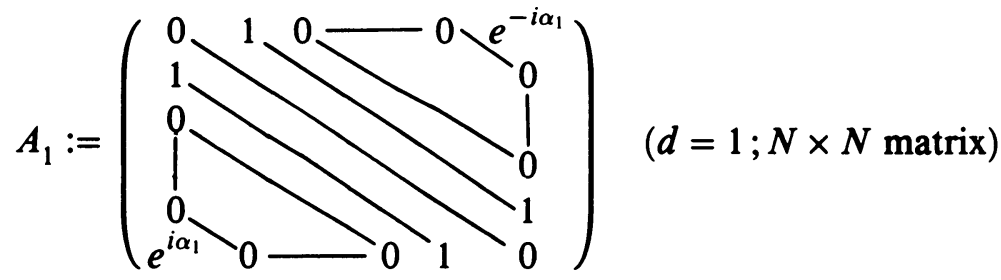

Received by the editors February 29, 1988.

1980 Mathematics Subject Classification (1985 Revision). Primary 35P99; Secondary 39A12. 
and<smiles>[H][Y10]#[Y10]N</smiles>

In this paper we prove the following results:

Theorem 1. Let $d \geq 2$ and $N \geq 2$ be integers and let $Q$ and $P$ be L-periodic, real valued potentials with the property that $\Delta_{\text {discrete }}+Q$ and $\Delta_{\text {discrete }}+P$ have the same spectrum when considered with the boundary condition (1.2) for any value $\alpha$ in $\mathbf{R}^{d}$. If $Q$ is of the form $Q(x)=\sum_{i=1}^{d} q_{i}\left(x_{i}\right)$, then $P$ is also of the form $P(x)=\sum_{i=1}^{d} p_{i}\left(x_{i}\right)$.

Theorem 2. Let $Q: \mathbb{Z}^{d} \rightarrow \mathbf{R}$ be a L-periodic potential with the property that $\Delta_{\text {discrete }}+Q$ and $\Delta_{\text {discrete }}$ have the same periodic spectrum, then $Q \equiv 0$.

Theorem 3. Let $N \geq 2$ and $d \geq 2$ be integers. Let two L-periodic potentials $Q$ and $P$ with values in $C$ have the property that $\Delta_{\text {discrete }}+Q$ and $\Delta_{\text {discrete }}+P$ have the same periodic spectrum. If both $Q$ and $P$ are of the form $Q\left(x_{1}, \ldots, x_{d}\right)=$ $\sum_{i=1}^{d} q_{i}\left(x_{i}\right)$ and $P\left(x_{1}, \ldots, x_{d}\right)=\sum_{i=1}^{d} p_{i}\left(x_{i}\right)$ with $\sum_{k=1}^{N} p_{i}(k)=0$ and $\sum_{k=1}^{N} q_{i}(k)=0 \quad(1 \leq i \leq d)$, then up to a permutation of the potentials $p_{i}$, the periodic spectrum of $D^{2}+p_{i}$ and $D^{2}+q_{i}$ are the same $(1 \leq i \leq d) . D^{2}$ denotes the discrete Laplacian in 1 - dim .

To put Theorem 1 and Theorem 2 into perspective let us recall two results from [K2]. To state them we need to introduce the following notation: Given a $L$-periodic potential $Q: \mathbb{Z}^{d} \rightarrow \mathbf{R}$ we denot by Iso $Q\left[\right.$ Iso $^{\text {Bloch }}(Q)$ ] the set of all $L$-periodic potentials $P: Z^{d} \rightarrow \mathbf{R}$ s.t. $\Delta_{\text {discrete }}+P$ and $\Delta_{\text {discrete }}+Q$ have the same periodic spectrum [the same spectrum when considered with the boundary conditions (1.2) for any vlaue of $\alpha$ ].

Result 1. \# Iso $Q \leq N^{d}$ ! and for $Q$ with pairwise different coefficients and $\sum_{x \in \Gamma} Q(x)^{2}$ sufficiently big \# Iso $Q=N^{d}$ !.

Result 2. For generic $Q$, Iso ${ }^{\text {Bloch }}(Q)=\left\{Q_{a},{ }^{r} Q_{a}: a \in \Gamma\right\}$ where $\Gamma:=\{x=$ $\left.\left(x_{1}, \ldots, x_{d}\right): 1 \leq x_{i} \leq N\right\}$ denotes a fundamental domain with respect to the lattice $L$, where $Q_{a}$ denotes the translated potential $Q_{a}(x):=Q(a+x)$ and where ${ }^{r} Q$ denotes the reflected potential ${ }^{r} Q(x)=Q(-x)$. For a periodic potential $Q: \mathbb{Z}^{d} \rightarrow \mathbf{R}$ of the form $Q\left(x_{1}, \ldots, x_{d}\right)=\sum_{i=1}^{d} q_{i}\left(x_{i}\right)$ denote by $I_{1}(Q)$ the set of $L$-periodic potentials $P: \mathbb{Z}^{d} \rightarrow \mathbf{R}$ of the form $P(x)=\sum_{i=1}^{d} p_{i}\left(x_{i}\right)$ s.t. $\Delta_{\text {discrete }}+Q$ and $\Delta_{\text {discrete }}+P$ have the same periodic spectrum. Then clearly, for $P$ in $I_{1}(Q)$, also $P_{a}$ and ${ }^{r} P_{a}(a$ in $\Gamma)$ are elements in $I_{1}(Q)$. By an 
elementary argument (cf. [K2]) one sees that $I_{1}(Q) \subseteq$ Iso $^{\text {Bloch }}(Q)$ for $Q$ 's of the form $Q(x)=\sum_{i=1}^{d} q_{i}\left(x_{i}\right)$.

Many of these potentials are nongeneric in the sense of Result 2 as the following counting off immediately reveals: For $Q(x)=\sum_{i=1}^{d} q_{i}\left(x_{i}\right)$ with pairwise different components and $q_{i}$ sufficiently big, one gets $\# I_{1}(Q)=d !(N !)^{d}$.

Thus the results presented in this paper are complementary to the ones given in [K2].

In the continuous analogue of (1.1), (1.2), assuming the lattice $L$ satisfies a certain generic condition, it follows from results of [ERT] (cf. also [MN]) that for a $L$-periodic real analytic potential $Q$ of the form $Q(x)=\sum_{i=1}^{d} q_{i}\left(x_{i}\right)$, any $L$-periodic real analytic potential $P$ with the property that $-\Delta+Q$ and $-\Delta+P$ have the same perioidic spectrum, must be of the form $P(x)=\sum_{i=1}^{d} p_{i}\left(x_{i}\right)$ s.t. $-d^{2} / d x_{i}^{2}+q_{i}$ and $-d^{2} / d x_{i}^{2}+p_{i}$ have the same periodic spectrum $(1 \leq i \leq d)$. To our astonishment it follows from [K2, Proposition] that the discrete analogue of this result cannot be true. This together with Theorem 3 indicates that the limit of the isospectral sets from the discrete to the continuous might be very subtle.

The paper is organized as follows: In $\S 2$, spectral invariants are derived using the discrete heat equation. These invariants are used in $\S 3$ to prove Theorem 1. Theorem 3 is proved in $\S 4$ by a combinatorial argument. To the best of our knowledge it is not known if Theorem 3 also holds in the continuous case.

Theorem 2 which has a continuous analogue follows directly from the heat invariant $\sum_{x \in \Gamma} Q(x)^{2}$, which is presented in $\S 2$. Clearly, the assumption in Theorem 2 that the potential $Q$ is real valued is essential for the proof. In the continuous case, at least in 1 dimension, the analogue of Theorem 2 is no longer true, if we consider also complex-valued potentials [GU] (cf. also [B1, B2]).

Notation is standard, except that in the sequel we denote by $\Delta$ rather than $\Delta_{\text {discrete }}$ the discrete Laplacian as defined above.

Acknowledgment. It is a great pleasure to thank Ira Gessel for helpful discussions.

\section{SPECTRAL INVARIANTS OF THE DISCRETE SCHRÖDINGER EQUATION}

For a $L$-periodic potiential $Q: \mathbf{Z}^{d} \rightarrow \mathbb{C}$ let us consider (1.1)-(1.2) with $\alpha=0$ and denote by $(\Delta+Q)_{\Gamma}$ the corresponding $\Gamma \times \Gamma$ matrix obtained by restricting $\Delta+Q$ to the fundamental domain $\Gamma$. Then the trace $\operatorname{Tr} \exp \left\{t(\Delta+Q)_{\Gamma}\right\}$ is a spectral invariant for each value of $t$. The first few coefficients of the expansion of this trace at $t=0$ can be easily computed. As a result we get

Proposition 2.1. $\sum_{x \in \Gamma} Q(x), \sum_{x \in \Gamma} Q(x)^{2}$ and $\sum_{x \in \Gamma} Q(x)^{3}$ are spectral invariants.

Let us now look at $\Delta+Q$ on the whole of $\mathbf{Z}^{d}$, denoting by $(\Delta+Q)(x, y)$ $\left(x, y\right.$ in $\left.z^{d}\right)$ its entries. Observe that each line and each column of $\Delta+Q$ has 
at most $(2 d+1)$ nonzero entries. Thus the products $(\Delta+Q)^{n} \quad(n \geq 0)$ of the infinite matrix $\Delta+Q$ are well defined. Define $M=\max \{1,|Q(x)|: x \in \Gamma\}$. By an inductive argument it is not hard to see that $\left|(\Delta+Q)^{n}(x, y)\right| \leq(2 d+1)^{n} M^{n}$ and thus $\sum_{n \geq 0}\left(t^{n} / n !\right)(\Delta+Q)^{n}(x, y)$ converges absolutely, together with the derivative $\sum_{n \geq 1}\left(n t^{n-1} / n !\right)(\Delta+Q)^{n}(x, y)$. We denote by $\exp t(\Delta+Q)$ the infinite sum $\sum_{n \geq 0}\left(t^{n} / n !\right)(\Delta+Q)^{n}$. For $\sum_{n \geq 0}\left(t^{n} / n !\right)(\Delta+Q)^{n}(x, y)$ we write simply $G(t, x, y):=: G(t, x, y, Q)$. Then $\partial_{t} G(t, \cdot, y)-(\Delta+Q) G(t, \cdot, y)=0$. Denote by $G_{L}(t, x, y, \alpha):=: G_{L}(t, x, y, \alpha, Q)$ the coefficients of the matrix $\exp t(\Delta+Q)_{\Gamma, \alpha}$ extended in $x$ and $y \quad \alpha$-periodically to the whole of $\mathbb{Z}^{d} \times$ $\mathbb{Z}^{d}$. $(\Delta+Q)_{\Gamma, \alpha}$ denotes the $\Gamma \times \Gamma$ matrix corresponding to $\Delta+\left.Q\right|_{\Gamma}$ when considered with $\alpha$-periodic boundary conditions. Then clearly $\partial_{t} G_{L}(t, \cdot, y, \alpha)-$ $(\Delta+Q) G_{L}(t, \cdot, y, \alpha)=0$ and $G_{L}(t, x+l, y, \alpha)=e^{2 \pi i \alpha \cdot l} G_{L}(t, x, y, \alpha)$ for any $l$ in $L$. By an inductive arugment one shows that $(\Delta+Q)^{n}(x, y)=0$ for

$$
\|x-y\|_{1}=\sum_{i=1}^{d}\left|x_{i}-y_{i}\right| \geq n+1 .
$$

From this it follows that $\sum_{l \in L} G(t, x+l, y)$ is absolutely convergent, together with the derivative $\sum_{l \in l} \partial_{t} G_{L}(t, x+l, y)$.

Finally, using the heat equation, one can show that $G_{L}(t, x, y, \alpha)=$ $\sum_{l \in L} e^{-2 \pi i \alpha^{*} l} G(t, x+l, y)$. Due to the fact that $\sum_{x \in \Gamma} G_{L}(t, x, x, \alpha)$ is an invariant of the Bloch spectrum for each value of $\alpha$ and of $t \geq 0$ we conclude from the formula above that $\sum_{x \in \Gamma} G(t, x+l, x)$ is an invariant of the Bloch spectrum for each value of $l$ in $L$ and all $t \geq 0$. Recall that $G(t, x, y)=$ $\sum_{n \geq 0}\left(t^{n} / n !\right)(\Delta+Q)^{n}(x, y)$ to conclude that $\sum_{x \in \Gamma}(\Delta+Q)^{n}(x+l, x)$ are invariants of the Bloch spectrum for all $l$ in $L$ and $n \geq 1$. For $e=\left(e_{1}, \ldots, e_{d}\right)$ in $\mathbf{Z}^{d}$ introduce the averaged potential $A_{e} Q(x):=\frac{1}{N} \sum_{m=1}^{N} Q(x+m e)$.

Proposition 2.2. Let $e$ be in $\mathbf{Z}_{+}^{d}$ with $\|e\|_{1}=1$. Then $\sum_{x \in \Gamma} A_{e} Q(x)^{2}$ is an invariant of the Bloch spectrum.

Proof. Let us consider $\sum_{x \in \Gamma}(\Delta+Q)^{N+2}(x+N e, x)$. Due to the fact that $\Delta^{n}(x, y)=0$ for $\|x-y\|_{1} \geq n+1$, we conclude that

$$
\begin{aligned}
\sum_{x \in \Gamma}(\Delta & +Q)^{N+2}(x+N e, x) \\
= & \sum_{x \in \Gamma} \Delta^{N+2}(x+N e, x)+(N+2) \sum_{x \in \Gamma} \Delta^{N+1}(x+N e, x) Q(x) \\
& +(N+2) \sum_{k=0}^{N} \sum_{\substack{x \in \Gamma \\
y \in \mathbf{Z}^{d}}} \Delta^{N-k}(x+N e, y) Q(y) \Delta^{k}(y, x) Q(x) .
\end{aligned}
$$


Clearly $\Delta^{N+1}(x+N e, x)$ is independent of $x$ and thus the second term on the right-hand side of the equation above is given by

$$
(N+2) \Delta^{N+1}(N e, 0) \sum_{x \in \Gamma} Q(x)
$$

which is a spectral invariant according to Proposition 2.1. One concludes that

$$
\sum_{k=0}^{N} \sum_{\substack{x \in \Gamma \\ y \in \mathbf{Z}^{d}}} \Delta^{N-k}(x+N e, y) Q(y) \Delta^{k}(y, x) Q(x)
$$

is an invariant of the Bloch spectrum. Use again that $\Delta^{n}(x, y)=0$ for $\|x-y\|_{1} \geq n+1$ to verify that

$$
\begin{aligned}
& \sum_{y \in \mathbf{Z}^{d}} \Delta^{N-k}(x+N e, y) Q(y) \Delta^{k}(y, x) Q(x) \\
& \quad=\Delta^{N-k}(x+N e, x+k e) Q(x+k e) \Delta^{k}(x+k e, x) Q(x) .
\end{aligned}
$$

By a straightforward inductive argument one sees that $\Delta^{k}(x+k e, x)=1$ for all $k \geq 0$ and $x$ in $\mathbf{Z}^{d}$, using $\|e\|_{1}=1$. Together with the fact that $\sum_{x \in \Gamma} Q(x)^{2}$ is a spectral invariant (Proposition 2.1) this shows that $\sum_{x \in \Gamma} \sum_{k=1}^{N} Q(x+k e) Q(x)$ is an invariant of the Bloch spectrum. The following computation finishes the proof of the proposition:

$$
\sum_{x \in \Gamma}\left(A_{e} Q\right)(x)^{2}=N \sum_{\substack{x \in \Gamma \\\langle x, e\rangle=0}}\left(A_{e} Q\right)(x)^{2}=\frac{1}{N} \sum_{x \in \Gamma} Q(x) \sum_{k=1}^{N} Q(x+k e)
$$

For the convenience of the reader we summarize the technical results about the discrete Laplacian which were used above with the following:

\section{Lemma 2.3.}

(1) $\Delta^{n}(x, y)=0$ for $x, y$ in $\mathbf{z}^{d}$ with $\|x-y\|_{1} \geq n+1$.

(2) $\Delta^{N+1}(x+N e, x)$ is independent of $x$ in $\mathbf{Z}^{d}$.

(3) $\Delta^{k}(x+k e, x)=1$ for $k \geq 1$ and $x$, e in $\mathbf{Z}^{d}$ with $\|e\|_{1}=1$.

\section{Proof of Theorem 1}

To prove Theorem 1 let us introduce the discrete Fourier transform. For an arbitrary potential $Q: \Gamma \rightarrow C$ we define its Fourier transform $\widehat{Q}$ to be

$$
\widehat{Q}(x)=\frac{1}{N^{d}} \sum_{k \in \Gamma} e^{-2 \pi i x \cdot k / N} Q(k)
$$

where $x \cdot k=\sum_{i=1}^{d} x_{i} k_{i}$. Then the inversion formula reads

$$
Q(x)=\sum_{k \in \Gamma} e^{2 \pi i x \cdot k / N} \widehat{Q}(k)
$$


and Parseval's identity takes the form

$$
\sum_{k \in \Gamma} Q(k) P(k)^{*}=N^{d} \sum_{k \in \Gamma} \widehat{Q}(k) \widehat{P}(k)^{*}
$$

where $*$ denotes complex conjugation. Denote by $e_{1}, \ldots, e_{d}$ a canonical basis in $\mathbf{R}^{d}$. For an arbitrary potential $Q: \Gamma \rightarrow \mathbb{C}$ introduce $(x$ in $\Gamma)$

$$
Q_{j}(x):=\sum_{\beta=1}^{N-1} e^{2 \pi i x_{j} \beta / N} \widehat{Q}\left(\beta e_{j}\right) \quad(1 \leq j \leq d)
$$

and

$$
Q_{d+1}(x):=\sum_{k \in \Gamma_{r}} e^{2 \pi i x \cdot k / N} \widehat{Q}(k)
$$

where $\Gamma_{r}:=\left\{k \in \Gamma \backslash\{(N, \ldots, N)\}: k \notin \beta e_{j}, 1 \leq \beta \leq N\right\}$. Then $Q(x)=$ $\widehat{Q}(N, \ldots, N)+\sum_{j=1}^{d+1} Q_{j}(x)$. Using Parseval's identity the following results, needed later, are easy to prove:

Lemma 3.1. For an arbitrary potential $Q: \Gamma \rightarrow \mathbb{C}$ the following properties hold:

(1) $\sum_{x \in \Gamma} Q_{j}(x) Q_{j^{\prime}}(x)^{*}=0 \quad\left(1 \leq j, j^{\prime} \leq d+1 ; j \neq j^{\prime}\right)$.

(2) $\sum_{x \in \Gamma}|Q(x)|^{2}=\sum_{j=1}^{d+1} \sum_{x \in \Gamma}\left|Q_{j}(x)\right|^{2}$.

(3) If $Q$ is real valued, so is $Q_{j} \quad(1 \leq j \leq d+1)$.

(4) $\sum_{\beta=1}^{N} Q_{j}\left(x+\beta e_{j}\right)=0$ ( $x$ in $\left.\Gamma ; 1 \leq j \leq d\right)$.

We now come to the proof of Theorem 1 . Let us assume that $Q$ is real valued and of the form $Q(x)=\sum_{i=1}^{d} q_{i}\left(x_{i}\right)$. By Proposition 2.1, $\sum_{x \in \Gamma} Q(x)$ is a spectral invariant. Without loss of generality we might assume that $\sum_{x \in \Gamma} Q(x)=$ 0 . Moreover we might assume that $\sum_{k=1}^{N} q_{j}(k)=0 \quad(1 \leq j \leq d)$. Thus $Q_{j}(x)=q_{j}\left(x_{j}\right)$ and $Q(x)=\sum_{j=1}^{d} Q_{j}(x)$. Similarly $P$ will be of the form $P(x)=\sum_{j=1}^{d+1} P_{j}(x)$. By Proposition 2.2

$$
\sum_{i=1}^{d} \sum_{x \in \Gamma} A_{e_{i}} Q(x)^{2}=\sum_{i=1}^{d} \sum_{x \in \Gamma} A_{e_{i}} P(x)^{2} .
$$

By Lemma 3.1(4) we have

$$
A_{e_{i}} Q(x)=\frac{1}{N} \sum_{\beta=1}^{N} Q\left(x+\beta e_{i}\right)=\sum_{\substack{1 \leq j \leq d \\ i \neq j}} Q_{j}(x)
$$

and

$$
A_{e_{i}} P(x)=\sum_{\substack{1 \leq j \leq d \\ j \neq i}} P_{j}(x)+A_{e_{i}} P_{d+1}(x) .
$$

By Lemma 3.1(2) one gets

$$
\sum_{x \in \Gamma}\left|A_{e_{i}} Q(x)\right|^{2}=\sum_{\substack{1 \leq j \leq d \\ j \neq i}} \sum_{\substack{x \in \Gamma \\ j}}\left|Q_{j}(x)\right|^{2}
$$


and

$$
\sum_{x \in \Gamma}\left|A_{e_{i}} P(x)\right|^{2}=\sum_{\substack{1 \leq j \leq d \\ j \neq i}} \sum_{x \in \Gamma}\left|P_{j}(x)\right|^{2}+\sum_{x \in \Gamma}\left|A_{e_{i}} P_{d+1}(x)\right|^{2} .
$$

Thus (3.1) can be rewritten as

$$
\begin{aligned}
& (d-1) \sum_{j=1}^{d} \sum_{x \in \Gamma} Q_{j}(x)^{2} \\
& =(d-1) \sum_{j=1}^{d} \sum_{x \in \Gamma} P_{j}(x)^{2}+\sum_{j=1}^{d} \sum_{x \in \Gamma} A_{e_{j}} P_{d+1}(x)^{2} .
\end{aligned}
$$

By Lemma 2.2

$$
(d-1) \sum_{x \in \Gamma} Q(x)^{2}=(d-1) \sum_{x \in \Gamma} P(x)^{2} .
$$

Subtract (3.2) from (3.3) and use Lemma 3.1(2) again to obtain

$$
0=(d-1) \sum_{x \in \Gamma} P_{d+1}(x)^{2}-\sum_{j=1}^{d} \sum_{x \in \Gamma}\left(A_{e_{j}} P_{d+1}(x)\right)^{2}
$$

or, using Parseval's identity,

$$
0=(d-1) \sum_{k \in \Gamma_{r}}|\widehat{P}(k)|^{2}-\sum_{j=1}^{d} \sum_{k \in \Gamma_{r}}\left|\left(A_{e_{j}} P\right)^{\wedge}(k)\right|^{2} .
$$

We will show that

$$
\sum_{j=1}^{d} \sum_{k \in \Gamma_{r}}\left|\left(A_{e_{j}} P\right)^{\wedge}(k)\right|^{2} \leq(d-2) \sum_{k \in \Gamma_{r}}|\widehat{P}(k)|^{2} .
$$

Clearly, Theorem 1 follows then from (3.4). To prove (3.5), write

$$
\begin{array}{r}
A_{e_{j}} P_{d+1}(x)=\frac{1}{N} \sum_{\beta=1}^{N} P_{d+1}\left(x+\beta e_{j}\right) \\
=\sum_{k \in \Gamma_{r}} e^{-2 \pi i k \cdot x / N} \widehat{P}(k) \frac{1}{N} \sum_{\beta=1}^{N} e^{-2 \pi i k_{j} \beta / N}
\end{array}
$$

to conclude that

$$
\sum_{k \in \Gamma}\left|A_{e_{j}} P(k)\right|^{2}=\sum_{k \in \Gamma_{r}}|\widehat{P}(k)|^{2}\left|\frac{1}{N} \sum_{\beta=1}^{N} e^{-2 \pi i k_{j} \beta / N}\right|^{2} .
$$

Now $\frac{1}{N} \sum_{\beta=1}^{N} e^{-2 \pi i k_{j} \beta / N}$ equals 1 , if $k_{j}=N$, and is 0 otherwise. But for $k \in \Gamma_{r}$ there exist $1 \leq j<j^{\prime} \leq d$ with $k_{j} \neq 0$ and $k_{j^{\prime}} \neq 0$. Thus

$$
\sum_{j=1}^{d}\left|\frac{1}{N} \sum_{\beta=1}^{N} e^{-2 \pi i k_{j} \beta / N}\right|^{2} \leq d-2 \quad\left(k \text { in } \Gamma_{r}\right)
$$


(3.6) together with (3.7) proves (3.5).

Remark. Similar arguments are used in [ERT] and [MN] to prove various results in the continuous case.

\section{Proof of TheOREM 3}

Let $Q: \mathbf{Z}^{d} \rightarrow \mathbb{C}$ be $L$-periodic and of the form $Q\left(x_{1}, \ldots, x_{d}\right)=\sum_{i=1}^{d} q_{i}\left(x_{i}\right)$. Let us denote by $\left(\lambda_{i k}\right)_{1 \leq k \leq N}$ the periodic eigenvalues of $D^{2}+q_{i}(1 \leq i \leq d)$, where $D^{2}$ denotes the discrete Laplacian in 1 dimension. Then the periodic eigenvalues of $\Delta+Q$ are all of the form $\lambda_{1 k_{1}}+\lambda_{2 k_{2}}+\cdots+\lambda_{d k_{d}}$ with $1 \leq k_{i} \leq N$ for $1 \leq i \leq d$. Theorem 3 claims that, up to permutation, the eigenvalues $\left(\lambda_{i k}\right)_{1 \leq k \leq N}$ for $1 \leq i \leq d$ can be recovered from the periodic eigenvalues of $\Delta+Q$, if we assume that $\sum_{k=1}^{N} \lambda_{i k}=0(1 \leq i \leq d)$. In order to state a general result from which Theorem 3 follows we need to introduce some notation. Let $N \geq 2$ and $d \geq 2$ be given. Denote the coordinates of $z$ in $\mathbb{C}^{N^{d}}$ by $z(k)$ where $k=\left(k_{1}, \ldots, k_{d}\right)$ is in $\Gamma:=\{1, \ldots, N\}^{d}$. Define $E$ to be the subspace of all points $z$ in $\mathbb{C}^{N^{d}}$ for which one can find sequences $\left(x_{i k}\right)_{1 \leq k \leq N, 1 \leq i \leq d}$ s.t. $z(k)=\sum_{i=1}^{d} x_{i k_{i}}$ for all $k$ in $\{1, \ldots, N\}^{d}$. Then $E$ is a linear subspace of dimension $d \cdot N-(d-1)$. Observe that $E$ can be described by the following relations: $z$ is in $E$ iff $z(k)+z(l)=z\left(k^{\prime}\right)+z\left(l^{\prime}\right)$ for all $k, l, k^{\prime}, l^{\prime}$ in $\Gamma$ with

$$
\bigcup_{i=1}^{d}\left\{k_{i}, l_{i}\right\}=\bigcup_{i=1}^{d}\left\{k_{i}^{\prime}, l_{i}^{\prime}\right\} \quad \text { (with multiplicities). }
$$

Now let us look at $\phi: \mathbb{C}^{N^{d}} \rightarrow \mathbb{C}^{N^{d}} / \Sigma_{\Gamma}, z \rightarrow[z]$ where $\Sigma_{\Gamma}$ denotes the symmetric group of the set $\Gamma$ and $[z]$ denotes the orbit of $z$ with respect to the natural action of $\Sigma_{\Gamma}$ on $\mathbb{C}^{N^{d}}$, i.e. permutation of the components of $Z$ in $C^{N^{d}}$. Introduce $\varphi:=\phi / E$. Then Theorem 3 follows from

Theorem 4.1. (1) For $z \in E, \varphi^{-1}\{\varphi(z)\}=H \cdot z$ where $H$ is the subgroup of $\Sigma_{\Gamma}$, leaving $E$ invariant.

(2) $H$ is the subgroup $G$ of $\Sigma_{\Gamma}$ generated by the permutations of the form

$$
\sigma\left(k_{1}, \ldots, k_{d}\right)=\left(\sigma_{1}\left(k_{1}\right), \ldots, \sigma_{d}\left(k_{d}\right)\right)
$$

and

$$
\sigma\left(k_{1}, \ldots, k_{d}\right)=\left(k_{\rho(1)}, \ldots, k_{\rho(d)}\right)
$$

where $\sigma_{1}, \ldots, \sigma_{d}$ are permutations in $\Sigma_{N}$, the symmetric group of the set $\{1, \ldots, N\}$, and $\rho$ is an arbitrary permutation of $\{1, \ldots, d\}$.

Remark. It follows from (2) that $\# H=d !(N !)^{d}$ and thus generically one gets $\# \varphi^{-1}\{\varphi(z)\}=d !(N !)^{d}$ by $(1)$.

Proof. (1) follows from the fact that $\Sigma_{\Gamma}$ is a finite group. Towards (2) let $\sigma$ be an element in $H$. Then $\sigma \cdot z$ is in $E$ for $z$ in $E$. Thus $\sigma \cdot z$ can be 
represented in the form

$$
\sigma \cdot z(k)=\sum_{i=1}^{d} x_{i k_{i}} \quad(k \in \Gamma)
$$

or

$$
z(k)=\sum_{i=1}^{d} x_{i \tau_{i}(k)}
$$

where $\tau(k)=\sigma^{-1}(k)=\left(\tau_{1}(k), \ldots, \tau_{d}(k)\right) \in \Gamma$. If necessary choose $\xi=$ $\left(\xi_{1}, \ldots, \xi_{d}\right)$ in $G$ where each $\xi_{i}$ is a permutation of $\{1, \ldots, N\}$ s.t. $\xi_{i} \circ$ $\tau_{i}((\beta, \ldots, \beta))=\beta \quad(1 \leq \beta \leq N ; 1 \leq i \leq d)$. So we might assume without any loss of generality that $\tau_{i}(\beta, \ldots, \beta)=\beta \quad(1 \leq \beta \leq N, 1 \leq i \leq d)$. Recall that elements in $E$ satisfy the following relations:

$$
\begin{array}{r}
z(k)+z(l)=z\left(k^{\prime}\right)+z\left(l^{\prime}\right) \text { for all } k, l, k^{\prime}, l^{\prime} \text { in } \Gamma \text { s.t. } \bigcup_{i=1}^{d}\left\{k_{i}, l_{i}\right\}=\bigcup_{i=1}^{d}\left\{k_{i}^{\prime}, l_{i}^{\prime}\right\} \\
\text { (with multiplicities). }
\end{array}
$$

Introduce $\theta$ to be the subset of $\Gamma$ consisting of $k=\left(k_{1}, \ldots, k_{d}\right)$ with $k_{i} \in$ $\{1,2\}$. Then for $k$ in $\theta$ we define $k^{*}$ to be $k^{*}=\left(3-k_{1}, \ldots, 3-k_{d}\right)$. Relation (4.1) implies that $\tau(k) \in \theta$ if $k$ is in $\theta$. As $\tau$ is $1-1$ this implies that $\tau(\theta)=\theta$. By interchanging $\tau_{1}, \ldots, \tau_{d}$, if necessary, we can assume without loss of generality that $\tau(k)=k$ for $k$ in $\theta$ by using (4.1) and the fact that $\tau$ is $1-1$. This is the case because a permutation of $\tau_{1}, \ldots, \tau_{d}$ corresponds to an element in $G$. Now (2) follows from

Lemma 4.2. Given $d \geq 2$, let $\tau: \Gamma \rightarrow \Gamma$ be 1-1 and have the following properties:

(i) $\tau((\beta, \ldots, \beta))=(\beta, \ldots, \beta)(1 \leq \beta \leq N)$,

(ii) $\tau(k)+\tau(l)=\tau\left(k^{\prime}\right)+\tau\left(l^{\prime}\right)$ $\left(k, l, k^{\prime}, l^{\prime} \in \Gamma\right.$ with $\bigcup_{i=1}^{d}\left\{k_{i}, l_{i}\right\}=\bigcup_{i=1}^{d}\left\{k_{i}^{\prime}, l_{i}^{\prime}\right\}$ (with multiplicities)),

(iii) $\tau(k)=k$ ( $k$ in $\theta)$.

Then $\tau$ is the identity.

Proof (by induction with respect to $d$ ). For $d=2$ one argues by induction with respect to $n$ in the following way. Let us assume that $\tau(k)=k$ for all $k=\left(k_{1}, k_{2}\right)$ in $\Gamma$ with $1 \leq k_{i} \leq n(i=1,2)$. We then have to show $\tau(k)=k$ for $k=\left(k_{1}, k_{2}\right)$ with $1 \leq k_{i} \leq n+1$. Choose $k:=(1,1), l:=(n+1, j)$, $k^{\prime}:=(i, j), l^{\prime}:=(n+1,1)$ and apply $(4.1)$ to conclude that $\tau_{1}(n+1, j)=$ $\tau_{1}(n+1,1)$ for $1 \leq j \leq n+1$. But $\tau_{1}(n+1, n+1)=n+1$ by assumption and thus $\tau_{1}(n+1, j)=n+1 \quad(1 \leq j \leq n+1)$. Moreover again by (4.1) one also concludes that $\tau_{2}(n+1, j)=j \quad(1 \leq j \leq n+1)$. Applying $(4.1)$ to $k=(1,1)$, $l=(i, n+1), k^{\prime}=(1, n+1), l^{\prime}=(i, 1)$ implies, arguing in the same way, that $\tau_{1}(i, n+1)=i \quad(1 \leq i \leq n+1)$ and $\tau(i, n+1)=n+1 \quad(1 \leq i \leq n+1)$. This proves Lemma 4.2 in the case $d=2$. Now let us assume that the statement of 
Lemma 4.2 holds up to dimension $d$; we then have to show it for $d+1$. But this follows easily from (4.1) and the fact that $\tau$ is by the induction hypothesis the identity on $\left\{\left(k_{1}, \ldots, k_{d}, 0\right): k_{i} \geq 1\right\}$.

\section{BIBLIOGRAPHY}

[B1] B. Birnir, Complex Hill's equation and the complex periodic KdV equations, Comm. Pure Appl. Math. 39 (1986), 1-49.

[B2] __ Singularities of the complex KdV flows, Comm. Pure Appl. Math. 39 (1986), 283-305.

[ERT] G. Eskin, J. Ralston and E. Trubowitz, On isospectral periodic potentials in $\mathbf{R}^{n}$, Comm. Pure Appl. Math. 37 (1984), 647-676.

[GU] V. Guillemin and A. Uribe, Spectral properties of a certain class of complex potentials, Trans. Amer. Math. Soc. 279 (1983), 759-771.

[K1] T. Kappeler, On isospectral potentials on a discrete lattice. I, Duke Math. J. 57 (1988), 135-150.

[K2] __, On isospectral potentials on a discrete lattice. II, Adv. Appl. Math. 9 (1988), 428-438

[LG] B. M. Levitan and M. G. Gasymov, Determination of a differential equation by two of its spectra, Russian Math. Surveys 19 (1964), 1-64.

[MN] S. A. Molchanov and M. V. Novitskii, Spectral invariants of the Schrödinger operator on the Euclidean torus, Soviet Math. Dokl. 33 (1986), 82-85.

Department of Mathematics, Brandeis University, Waltham, Massachusetts 02254

Current address: Department of Mathematics, Brown University, Providence, Rhode Island 02912 